\title{
The nutritional status and outcome of patients admitted to an intestinal failure unit
}

\author{
A. Culkin \\ Lennard-Jones Intestinal Failure Unit, St Mark's Hospital, Harrow, HA1 3UJ, UK
}

Intestinal failure (IF) is defined as "reduced gastrointestinal absorption to the extent that macronutrient and/or fluid supplements are required". Depending on the severity of IF, patients can be treated with nutrition and fluid via the oral, enteral or parenteral route) ${ }^{(1)}$. Malnutrition ocurs in IF due to the underlying condition plus complications including sepsis and infection and is associated with an increased length of stay, reduced response to treatment, higher risk of compliations and increased mortality ${ }^{(2)}$. The aim of this study was to assess the nutritional status of patients transfered to an intestinal failure unit (IFU) during a 12 month period and document any changes in the provision of artificial nutrition.

Height and weight were measured and body mass index (BMI) was calculated. Mid-arm circumference (MAC) and tricep skinfold thickness (TST) were measured to calculate mid-arm muscle circumference (MAMC) and compared to published standards ${ }^{(3)}$. Percentage weight loss over the past three to six months was estimated from patient recall. Handgrip was measured to assess functional capacity and compared to published standards ${ }^{(4)}$.

Eighty one patients were admitted to the IFU and seventy two were assessed as nine patients died. There were 39 males and 38 females with a mean age of $53 \pm 15.4$ years. The causes of IF included short bowel $(n=28)$, fistula $(n=27)$, small bowel obstruction $(n=10)$, malabsorption $(n=5)$ and others $(n=2)$. Route of nutrition on transfer included parenteral $(n=46)$, enteral $(n=5)$ and oral $(n=21)$. The mean $\%$ weight loss was $9.4 \pm 10.5 \%$ (range $0-35 \%$ ). MAC was $<5$ th centile in 57 patients (39\%), TST was $<5$ th centile in 10 patients $(18 \%)$ and MAMC was <5th centile in 16 patients (29\%). Handgrip was measured and was below $85 \%$ of normal in $83 \%$ of patients $(n=59)$. According to the NICE definition of malnutition ${ }^{(5)}, 58 \%$ of patients were malnourished on transfer. It can be seen from the table that patients maintained nutritional status during their in patient stay with a trend towards an improvement in handgrip strength. A significant reduction in energy and nitrogen from PN was observed due to the introduction of oral feeding and weaning from PN.

\begin{tabular}{|c|c|c|c|}
\hline Parameter & Admission $($ Mean \pm SD) & Discharge (Mean $\pm \mathrm{SD})$ & Difference ( $p$ value) \\
\hline Weight (kg) & $63.6 \pm 17.1$ & $63.6 \pm 14.9$ & 0.4 \\
\hline BMI $\left(\mathrm{kg} / \mathrm{m}^{2}\right)$ & $22.7 \pm 5.5$ & $22.7 \pm 5.5$ & 0.4 \\
\hline TST (mm) & $14.4 \pm 6.6$ & $14.4 \pm 5.7$ & 0.49 \\
\hline MAMC (cm) & $22.9 \pm 3.6$ & $23.2 \pm 3.0$ & 0.35 \\
\hline Handgrip (kg) & $19.4 \pm 8.5$ & $22.0 \pm 7.2$ & 0.06 \\
\hline Energy from PN (kcal) & $1132 \pm 811$ & $717 \pm 754$ & $<0.0001$ \\
\hline Nitrogen from PN $(\mathrm{g})$ & $8 \pm 5.6$ & $5.9 \pm 6.9$ & 0.01 \\
\hline
\end{tabular}

From the results obtained it is clear that patients presenting to an IFU are malnourished with a poor functinal capacity despite the majority receiving parenteral nutrition. This may have been due to poor provision of PN and catheter relatated sepsis before transfer ${ }^{(6)}$. It is important that these complex patients are cared for by experienced nutrition teams in order to minimise the risk of malnutition and PN complications.

1. Nightingale JMD (2001) Intestinal Failure. London, Greenwich Medical Media Ltd. pp. xix-xx.

2. Stratton RJ, Green CJ \& Elia M (2003) Disease related malnutrition: an evience based approach to treatment. Wallingford, UK. CABI publishing.

3. Bishop CW, Bowen PE \& Ritchey SJ (1981) Am J Clin Nutr 34, 2530-2539.

4. Klidjian AM, Archer TJ, Foster KJ \& Karran SJ (1982) JPEN 6, 119-121.

5. National collaborating centre for acute care, February 2006. Nutrition support in adults. Oral nutrition support, enteral tube feeding and parenteral nutrition. ISBN 0-9549760-2-9.

6. http://www.ncepod.org.uk/2010report1/downloads/PN_report.pdf. 